\title{
Ensayo Le Chatelier-Anstett Influencia del tiempo y modo de hidratación (I)
}

J. L. SAGRERA

Dr. en Ciencias Químicas

\section{O. INTRODUCCION}

Dentro del ensayo de sulfatodurabilidad, aplicando el método de Le Chatelier-Anstett, (1) se estudia en el presente artículo la variación de resultados aplicando dos normas de preparación de probetas, que difieren en la duración y modo de hidratación. (2)

Los resultados se refieren a la variación dimensional de las probetas, y a la dureza de las mismas, medida con la aguja de Vicat.

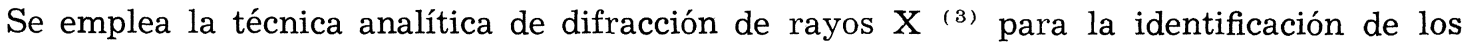
compuestos cristalinos formados tanto en la hidratación previa de los cementos, como durante el tiempo, 180 días, en que actúa el agresivo $\left(\mathrm{SO}_{4} \mathrm{Ca} .2 \mathrm{H}_{2} \mathrm{O}\right)$ sobre ellos.

\section{CEMENTOS ESTUdiados}

Este estudio comprende los resultados obtenidos con el ensayo de Le Chatelier-Anstett aplicado a los tipos de cemento que a continuación se detallan:

\begin{tabular}{|c|c|c|}
\hline Cemento & Denominación Internacional & Denominación Española \\
\hline n. ${ }^{\circ} 1$ & $\mathrm{OPC}$ & $\mathrm{P}$ \\
\hline n. ${ }^{\circ} 2$ & SRPC & PAS \\
\hline n. ${ }^{\circ} 3$ & $\mathrm{PZC}$ & PUZ \\
\hline n. ${ }^{\circ} 4$ & PZC sp. & PAS-PUZ \\
\hline n. ${ }^{\circ} 5$ & $\mathrm{BLC}$ & PHA \\
\hline
\end{tabular}

\section{ANALISIS QUIMICO DE LOS CEMENTOS ESTUDIADOS}

\begin{tabular}{|c|c|c|c|c|c|}
\hline Cementos & 1 & 2 & 3 & 4 & 5 \\
\hline $\mathrm{SiO}_{2}$ & 20,87 & 21,21 & 19,73 & 31,90 & 29,30 \\
\hline R.I. & 0,10 & 0,04 & 13,60 & 5,08 & 0,38 \\
\hline $\mathrm{Al}_{2} \mathrm{O}_{3}$ & 5,24 & 3,75 & 9,99 & 3,02 & 11,70 \\
\hline $\mathrm{Fe}_{2} \mathrm{O}_{3}$ & 2,80 & 4,13 & 3,45 & 3,13 & 1,04 \\
\hline $\mathrm{CaO}$ & 63,87 & 64,44 & 43,51 & 47,94 & 45,85 \\
\hline $\mathrm{MgO}$ & 1,36 & 1,14 & 1,37 & 0.88 & 4,91 \\
\hline $\mathrm{SO}_{3}$ & 3,45 & 2,74 & 1,95 & 2,99 & 2,83 \\
\hline P.F. & 1,10 & 1,32 & 3,13 & 4,39 & 0,95 \\
\hline $\mathrm{K}_{2} \mathrm{O}$ & 0,86 & 0,94 & 2,46 & 0,71 & 0,88 \\
\hline $\mathrm{Na}_{2} \mathrm{O}$ & 0,37 & 0,21 & 0,69 & 0,16 & 0,74 \\
\hline
\end{tabular}




\section{AGRESIVO EMPLEADO}

Así como en otra publicación ${ }^{(4)}$ de la División de Durabilidad se han empleado dos tipos de agresivo $\left(\mathrm{SO}_{4} \mathrm{Ca} \cdot 2 \mathrm{H}_{2} \mathrm{O}\right.$ y $\left.\mathrm{SO}_{4} \mathrm{Na}_{2} \cdot 10 \mathrm{H}_{2} \mathrm{O}\right)$, en este trabajo sólo se han preparado probetas utilizando $\mathrm{SO}_{4} \mathrm{Ca} \cdot 2 \mathrm{H}_{2} \mathrm{O}$.

Con objeto de comprobar la pureza de dicho agresivo se han llevado a cabo análisis cuantitativos por difracción de rayos $\mathrm{X}$ de la muestra, comprobándose la ausencia de otros compuestos cristalinos que no sean $\mathrm{SO}_{4}$ Ca. $2 \mathrm{H}_{2} \mathrm{O}$.

\section{PREPARACION De LAS PROBETAS}

\subsection{Modo de hidratación del cemento}

Referente al modo de hidratación del cemento hay dos variantes que en la División de Durabilidad del Instituto "Eduardo Torroja" se han denominado: Norma Francesa y Norma Acelerada; cada una de ellas consta de los siguientes pasos:

\subsubsection{Norma Francesa}

El cemento se muele hasta pasar por un tamiz de 4.900 mallas.

Dos partes de cemento se amasan con una de agua.

La mezcla se mantiene en humedad saturada durante 24 horas.

Se trocea y sumerge bajo agua en una caja de plástico con tapa del mismo material durante 200 días.

El cemento así hidratado se trocea a tamaño de $5 \mathrm{~mm}$.

Se deseca en estufa a $40^{\circ} \mathrm{C}$ durante 17 días.

Dos partes de este cemento hidratado se mezclan con una parte de $\mathrm{SO}_{4} \mathrm{Ca} .2 \mathrm{H}_{2} \mathrm{O}$.

La mezcla se pulveriza hasta pasar por un tamiz de $0,2 \mathrm{~mm}$.

A la mezcla se le añade un $6 \%$ en peso de agua, y en molde cilíndrico y a una presión de $5 \mathrm{kp} / \mathrm{cm}^{2}$ se hace la probeta.

\subsubsection{Norma acelerada}

Dentro de una caja cilíndrica de acero inoxidable con tapa del mismo material, se introducen $500 \mathrm{~g}$ de cemento anhidro, $1.000 \mathrm{~cm}^{3}$ de agua filtrada y $1.400 \mathrm{~g}$ de bolas de acero perfectamente limpias. Sobre una máquina agitadora, se mantienen en continuo movimiento cada uno de los cementos estudiados hasta cumplir los tiempos siguientes:

\begin{tabular}{|c|c|}
\hline Cemento & Tiempo de Hidratación \\
\hline n. 1 & 72 horas \\
\hline n. ${ }^{\circ} 2$ & $96 "$ \\
\hline n. ${ }^{0} 3$ & 120 \\
\hline n. ${ }^{\circ} 4$ & 72 \\
\hline n. 5 & 216 \\
\hline
\end{tabular}

Estos tiempos de hidratación para cada cemento han sido determinados por análisis cualitativo de difracción de rayos $\mathrm{X}$, usando tomas de muestra a diferentes tiempos de agi- 
tación y considerando acabado dicho tiempo, al aparecer en los análisis un pico identificado como monosulfoaluminato tetracálcico hidratado. (2)

\section{ANALISIS CUALITATIVO POR DIFRACCION DE RAYOS $\mathbf{x}$}

El equipo utilizado para la determinación cualitativa de los compuestos cristalinos, presentes en cada una de las etapas por las que pasan los cementos estudiados - hidratación, desecación y ataque-, es marca Philips tipo PW-1010 con unidad de registro PW-1540; las condiciones de trabajo aplicadas son:

Anodo de cobre trabajando a $40 \mathrm{kV}$ y $24 \mathrm{~mA}$.

Rendijas de colimación: $1^{\circ}-0,2-1^{\circ}$.

Factores de escala: 8-2-1.

Velocidad de la carta: 40.

Contador proporcional: $1.650 \mathrm{~V}$.

Velocidad de registro: $2^{\circ}$ por minuto.

Las determinaciones cualitativas se han aplicado a las muestras de los cementos hidratados, según Norma Francesa; a las tomas hechas a diferentes tiempos de los cementos hidratados, según Norma Acelerada; a los compuestos cristalinos formados en las muestras de cemento hidratado, según Norma Francesa, después de permanecer 17 días en estufa a $40^{\circ} \mathrm{C}$; a la identificación del compuesto monosulfoaluminato tetracálcico hidratado y su comprobación mediante cambios de su agua de cristalización.

\section{CARACTERISTICAS FISICAS DE LAS PROBETAS}

Sobre cada una de las probetas, y a las edades inicial y final del ensayo, se han tomado medidas de diámetro, altura y penetrabilidad de la aguja de Vicat, con el fin de comprobar las variaciones que cada cemento sufre en función del modo y tiempo de hidratación.

Finalmente, y como estudio comparativo, se calculan los incrementos, en tanto por ciento, de los diámetros y alturas que cada una de las probetas han sufrido, para, de esta manera, poder sacar conclusiones sobre la sulfatodurabilidad de los cementos, aplicando el método de Le Chatelier-Anstett con dos diferentes tipos de preparación de probetas.

También se facilitan fotos de algunas probetas atacadas cuyo estado externo ha sufrido variaciones, como son: grietas, fisuras, escamas, hinchamientos, etc.

\section{\%. RESULTADOS OBTENIDOS}

Los resultados obtenidos se agrupan por cementos, ya que el presente trabajo se realiza para estudiar la influencia que tiene el modo y tiempo de hidratación de cada cemento sobre su durabilidad al ataque del sulfato cálcico dihidrato.

Los números adoptados a la izquierda de cada compuesto determinado en las gráficas de difracción de rayos $\mathrm{X}$ son arbitrarios, pero directamente proporcionales a la cantidad del compuesto al que pertenecen. 


\subsection{Cemento n. ${ }^{\circ} 1$}
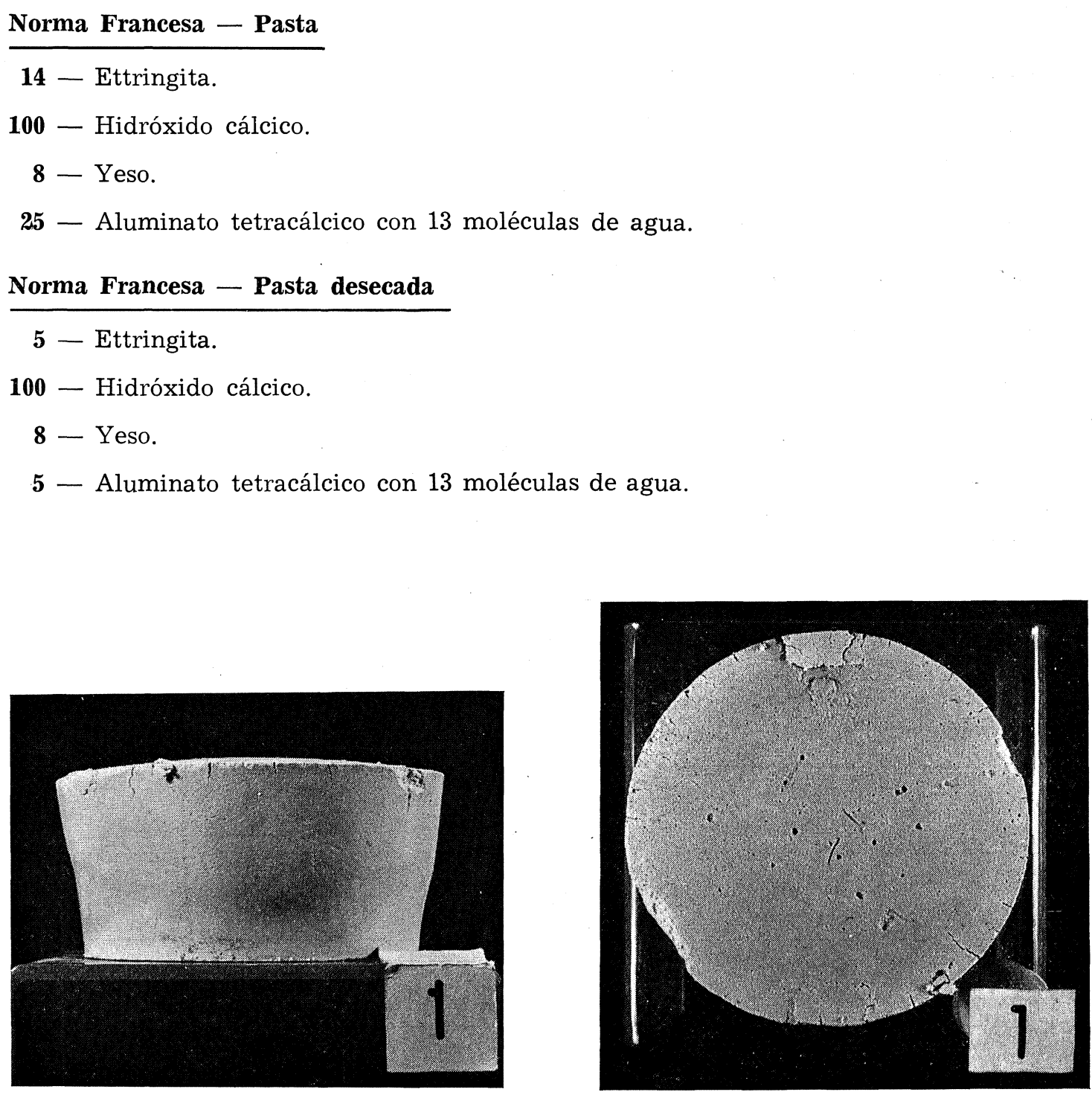

Cemento n.o 1 - Norma Francesa

Comentario: Como puede verse por los contenidos de cada compuesto, la ettringita disminuye de 14 a 5 debido a la posible pérdida de agua por calentamiento en estufa durante un período tan largo, aunque la temperatura sea baja $\left(40^{\circ} \mathrm{C}\right)$. Según Mehta ${ }^{(5)}$, el tratamiento térmico prolongado descompone la ettringita y no vuelve a formarse, excepto en el caso de una rehidratación con humedades relativas por encima del $90 \%$.

Lo mismo ocurre con el aluminato tetracálcico con 13 moléculas de agua, que pasa de 25 a 5 unidades. En cambio, el yeso no pierde concentración en la muestra de pasta desecada y lo mismo le ocurre al hidróxido cálcico, debido a la poca influencia de esa temperatura. 
16 - Ettringita.

100 - Hidróxido cálcico.

57 - Monosulfoaluminato tetracálcico con 12 moléculas de agua.
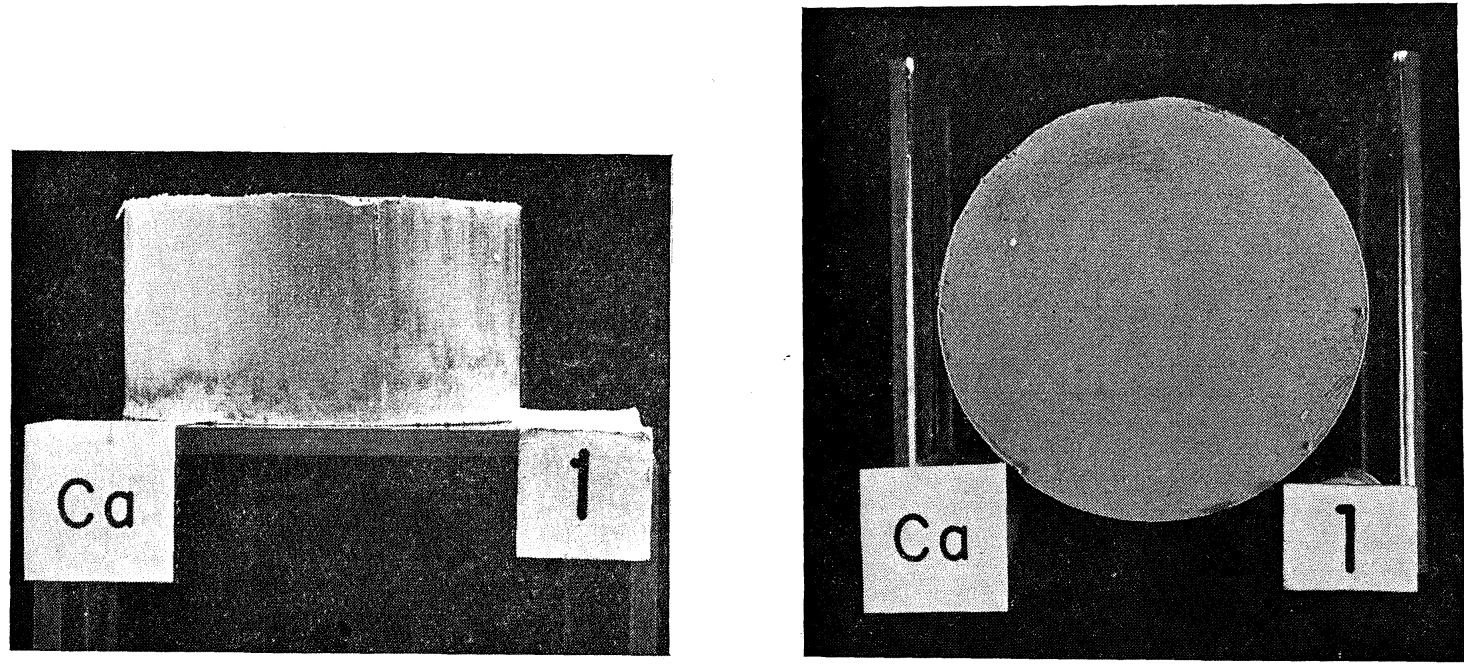

Cemento n.o 1 - Norma Acelerada

Comentario: Comparando estos resultados con los de la Norma Francesa - Pasta, puede verse que con la Norma Acelerada se elimina el yeso. Según Mehta (5), el yeso se detecta mediante difracción de rayos $\mathrm{X}$ en los primeros momentos de hidratación, dado que la Norma Acelerada acorta estos tiempos, el compuesto yeso se ha formado en las primeras horas y, por lo tanto, a las 72 horas de hidratación ha desaparecido del diagrama de rayos X. También vemos que ha desaparecido el aluminato tetracálcico hidratado con 13 moléculas de agua. Chatterji (6) afirma que todo el aluminato tetracálcico con 13 moléculas de agua de un cemento hidratado, si está en presencia de iones $\mathrm{SO}_{4}{ }^{2}$, pasa a formar monosulfoaluminato tetracálcico con 12 moléculas de agua siempre y cuando en el sistema exista $\mathrm{Ca}(\mathrm{OH})_{2}$, compuestó que, como puede verse, aparece en la Norma Acelerada - Pasta.

Las cantidades de ettringita e hidróxido cálcico no varían de una norma a otra.

\section{Resultados sobre probetas}

Según se indica en el apartado 6, a continuación se detallan los resultados obtenidos de las medidas del diámetro, altura y penetrabilidad de la aguja de Vicat:

\begin{tabular}{|c|c|c|c|c|c|c|c|c|}
\hline \multirow{2}{*}{ NORMA } & \multicolumn{3}{|c|}{ DIAMETROS } & \multicolumn{3}{c|}{ ALTURAS } & \multicolumn{2}{c|}{ VICAT } \\
\cline { 2 - 8 } & Inicial & Final & $\Delta \%$ & Inicial & Final & $\Delta \%$ & Inicial & Final \\
\hline Francesa & 80,2 & 95,2 & 18,7 & 37,5 & 45,3 & 20,8 & 1 & 40 \\
Acelerada & 80,2 & 81,1 & 1,1 & 43,4 & 43,8 & 1,6 & 0 & 0 \\
\hline
\end{tabular}


Como demostración del estado externo de las probetas, según la norma de preparación, se facilitan fotos de vista lateral y vertical de algunas de ellas. A simple vista, y por comparación, se comprueba la influencia que tienen las dos diferentes normas de preparación para cada uno de los cementos estudiados.

\subsection{Cemento n. $^{\circ} 2$}

$$
\begin{aligned}
& \text { Norma Francesa - Pasta } \\
& \mathbf{8} \text { - Ettringita. } \\
& \mathbf{1 0 0} \text { - Hidróxido cálcico. }
\end{aligned}
$$

\section{Norma Francesa - Pasta desecada}

100 - Hidróxido cálcico.
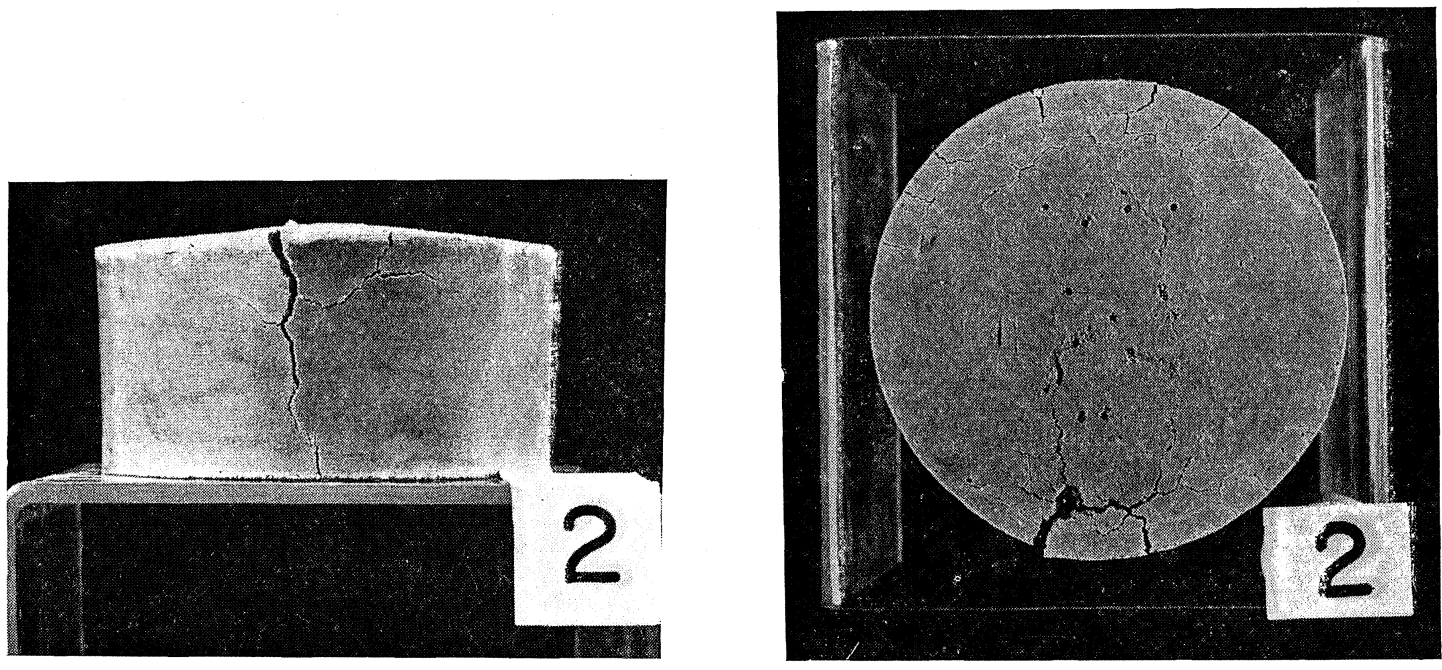

Cemento n.o 2 - Norma Francesa

Comentario: El único compuesto cristalino que permanece inalterado es el hidróxido cálcico, ya que la baja temperatura de desecación no influye sobre su cuantía ni estado. La poca cantidad de ettringita en la Norma Francesa - Pasta, y su mal estado de cristalización se debe a la poca cantidad de aluminato tricálcico presente en este cemento debido a su condición de cemento PAS. La ausencia de ettringita en la Norma Francesa - Pasta desecada se debe, según Kantro y Copeland (7), a la baja cuantía procedente ya de la Norma Francesa - Pasta que al ser desecada, desaparece, salvo humedecer la muestra por encima del $90 \%$ de humedad relativa.

\section{Norma Acelerada - Pasta}

$$
\begin{aligned}
& 15 \text { - Ettringita. } \\
& \mathbf{1 0 0} \text { - Hidróxido cálcico. } \\
& \mathbf{4 7} \text { - Monosulfoaluminato tetracálcico con } 19 \text { moléculas de agua. }
\end{aligned}
$$



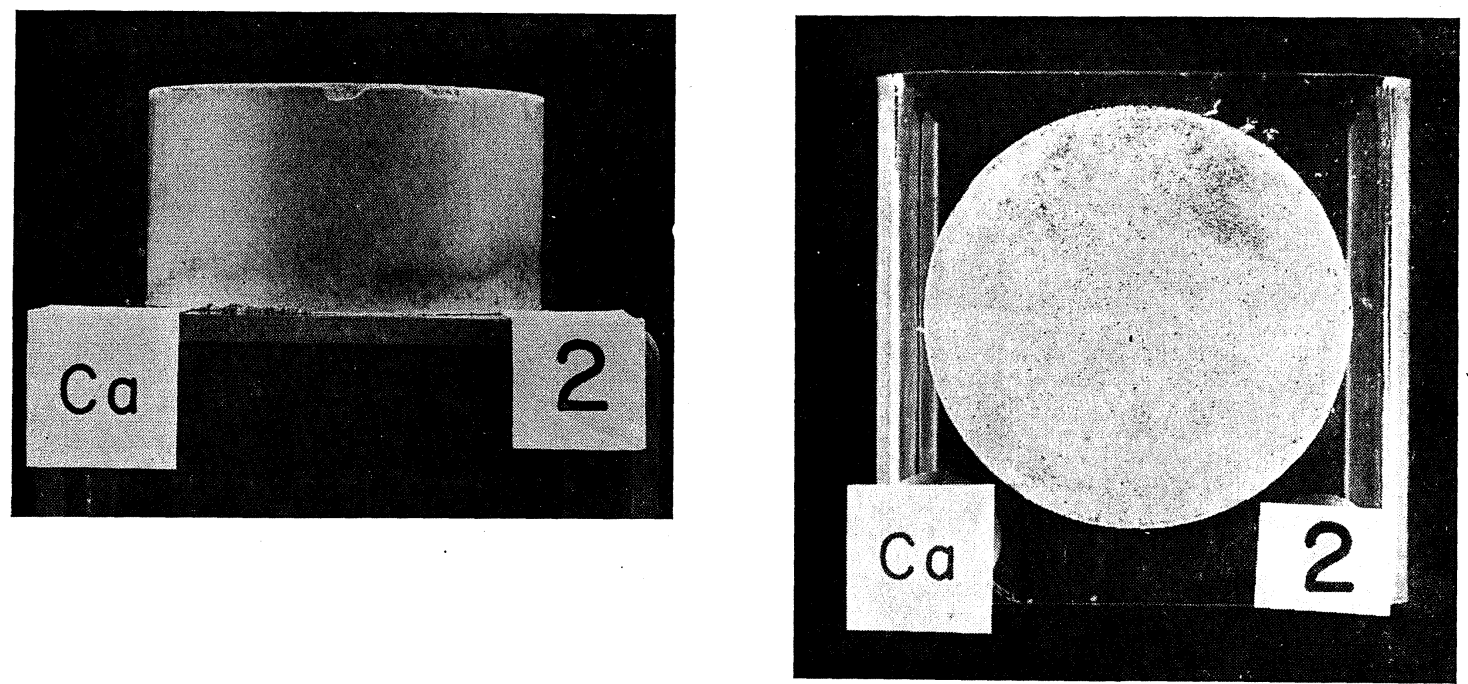

Cemento n.o 2 - Norma Acelerada

Comentario: Tanto la cantidad de ettringita como la de hidróxido cálcico son superiores en la Norma Acelerada; además la ettringita está bien cristalizada debido al factor tiempo de hidratación (96 horas) y, sobre todo, a la agitación que acelera el proceso. Hay un nuevo compuesto en la Norma Acelerada, conocido como monosulfoaluminato tetracálcico con 19 moléculas de agua, o sea, el estado máximo de hidratación de este compuesto. Según Turriziani ${ }^{(8)}$, cuando la relación agua/cemento es mayor o igual a 10, ya al cabo de 24 horas de hidratación con agitación puede llegarse a fases que contienen 19 ó 20 moléculas de agua.

\section{Resultados sobre probetas}

\begin{tabular}{|c|c|c|c|c|c|c|c|c|c|}
\hline \multirow{2}{*}{ NORMA } & \multicolumn{3}{|c|}{ DIAMETRos } & \multicolumn{2}{c|}{ ALTURAS } & \multicolumn{3}{c|}{ VICAT } \\
\cline { 2 - 7 } & Inicial & Final & $\Delta \%$ & Inicial & Final & $\Delta \%$ & Inicial & Final \\
\hline Francesa & 80,2 & 81,7 & 1,87 & 37,0 & 37,6 & 1,62 & 0 & 0 \\
Acelerada & 80,2 & 80,6 & 0,50 & 43,4 & 43,7 & 0,70 & 0 & 0 \\
\hline
\end{tabular}

Del mismo modo que del cemento n. ${ }^{\circ} 1$, se facilitan fotos del estado externo de las probetas.

\section{y.3. Cemento n. ${ }^{\circ} 3$}

\section{Norma Francesa - Pasta}

28 - Yeso.

29 - Aluminato tetracálcico con 13 moléculas de agua. 
Norma Francesa - Pasta desecada

29 - Hidróxido cálcico.

40 - Yeso.

100 - Aluminato tetracálcico con 13 moléculas de agua.

18 - Monosulfoaluminato tetracálcico con 12 moléculas de agua.
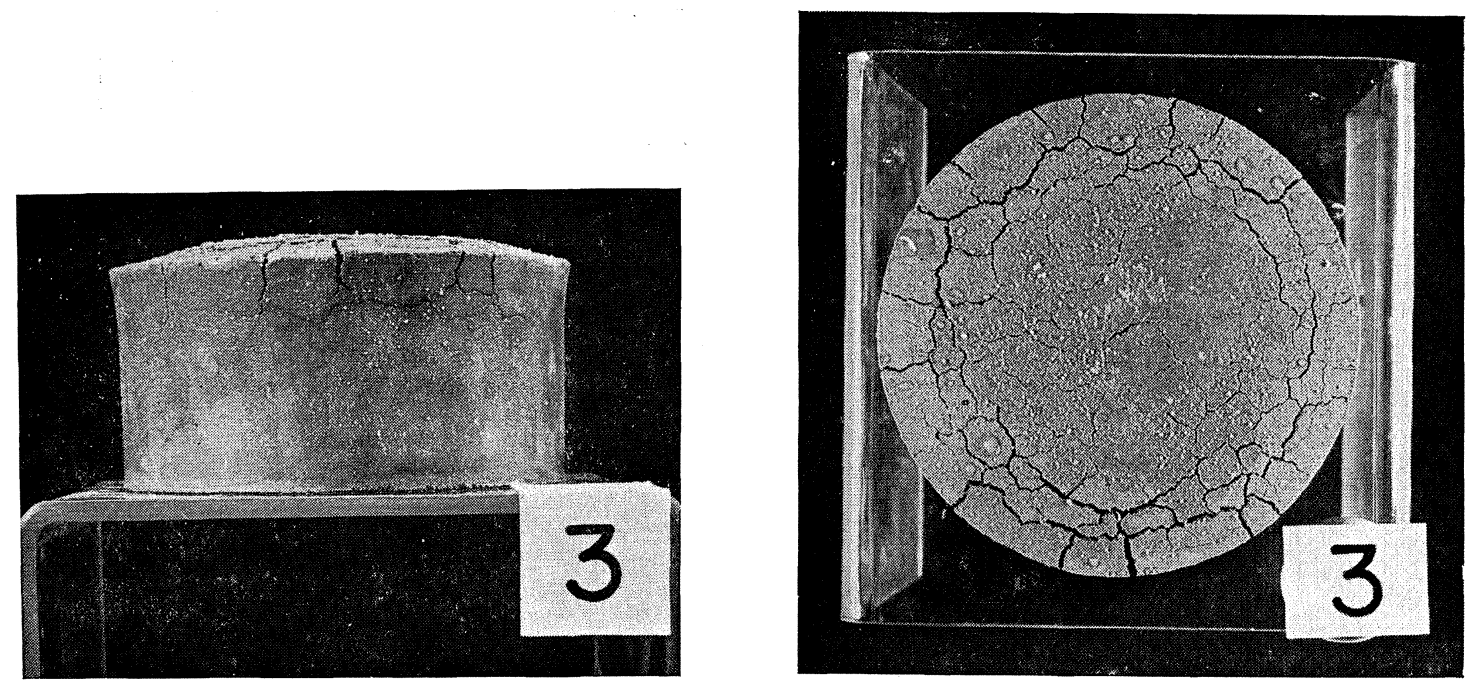

Cemento n.o 3 - Norma Francesa

Comentario: La aparición de $\mathrm{Ca}(\mathrm{OH})_{2}$ en la pasta desecada que, como puede verse, no existe en la pasta, parece a primera vista ser motivada por un posible estado inicial no cristalino y, por tanto, no detectado por difracción de rayos $\mathrm{X}$, pero que, en el transcurso del tiempo (17 días) y con aumento de temperatura (desde $20^{\circ} \mathrm{C}$ ambientales a $40^{\circ} \mathrm{C}$ de estufa), puede evolucionar hacia la forma cristalina, siendo detectada en la pasta desecada. Bien es verdad que este mismo argumento podía haberse aplicado a los otros cementos estudiados ( $\mathrm{P}$ y PAS), ya que han pasado por iguales estados de hidratación y desecación en estufa. Por ello, puede aplicarse otra hipótesis al caso de Norma Francesa - Pasta desecada: que la ausencia de $\mathrm{Ca}(\mathrm{OH})_{2}$ en la gráfica de difracción de rayos X se debe a un enmascaramiento del $\mathrm{Ca}(\mathrm{OH})_{2}$, debido a la puzolana. En una próxima publicación se darán los resultados de la aplicación de la experiencia inductiva siguiente:

a) Hidratación con cemento puzolánico.

b) Toma de tres muestras para desecar la primera a $40^{\circ} \mathrm{C}$; la segunda para desecar a $80^{\circ} \mathrm{C}$ y la tercera para dejar evolucionar preservándola del $\mathrm{CO}_{2}$ ambiental.

De cada una de estas tres muestras, y a edades iguales, se harán diagramas de rayos X, comprobando en ellos: la posible aparición de hidróxido cálcico, no sólo en las desecadas a $40^{\circ}$ y a $80^{\circ} \mathrm{C}$, sino en la preservada de $\mathrm{CO}_{2}$, lo cual confirmará que el paso al estado cristalino del hidróxido cálcico es sólo función del tiempo.

También puede ocurrir, que la cantidad de hidróxido cálcico detectado en la muestra de- 
secada a $80^{\circ} \mathrm{C}$ sea mayor que a $40^{\circ} \mathrm{C}$, lo cual demostrará que la formación del hidróxido cálcico cristalino también es función de la temperatura.

Otros hechos que parecen confirmar que la temperatura influye en la formación de compuestos, es el aumento en la cantidad de aluminato tetracálcico con 13 moléculas de agua de 29 a 100, así como la aparición de monosulfoaluminato tetracálcico con 12 moléculas de agua en pasta desecada, ya que la formación de este compuesto, según Seligmann y Greening (9) y Chatterji ${ }^{(10)}$, se da cronológicamente después del aluminato tetracálcico con 13 moléculas de agua en los procesos de hidratación.

\section{Norma Acelerada - Pasta}

$$
12 \text { - Ettringita. }
$$

100 - Hidróxido cálcico.

15 - Aluminato tetracálcico con 13 moléculas de agua.

100 - Monosulfoaluminato tetracálcico con 18 moléculas de agua.
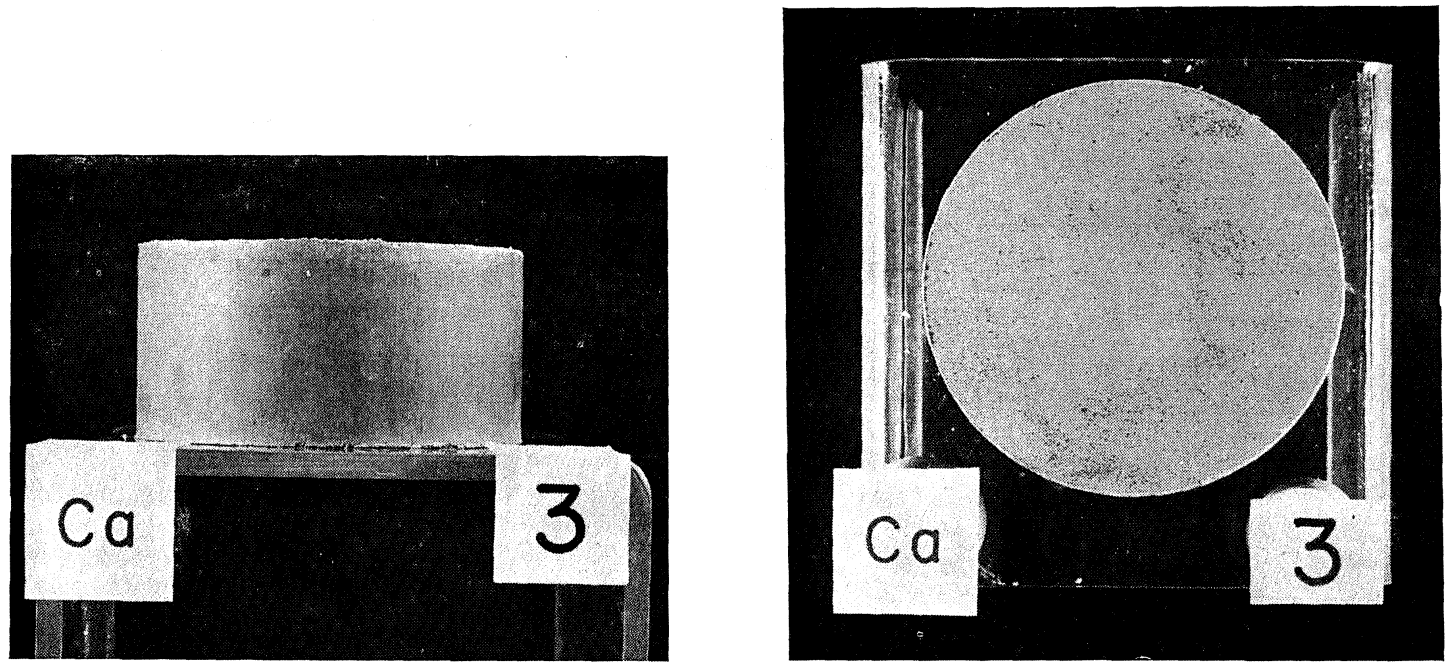

Cemento n.o 3 - Norma Acelerada

Comentario: Por comparación con la Norma Francesa - Pasta puede comprobarse la desaparición de yeso, a causa de que este compuesto, según Seligmann y Greening (9), se detecta en los primeros momentos de la hidratación, y al corresponder la gráfica a la Norma Acelerada - Pasta (120 horas de hidratación) ya ha desaparecido.

En cambio, la gráfica correspondiente a Norma Acelerada - Pasta posea ettringita que no había en la Norma Francesa - Pasta debido a la baja hidratación del cemento.

La cantidad de alúminato tetracálcico con 13 moléculas de agua se ha reducido a casi la mitad, como consecuencia probablemente de la formación de monosulfoaluminato tetracálcico con 18 moléculas de agua, cuya formación se realiza, según Chatterji (6), a partir del aluminato tetracálcico con 13 moléculas de agua y la debida proporción de iones $\mathrm{SO}_{4}{ }^{\prime}$. 


\section{Resultados sobre probetas}

\begin{tabular}{|c|c|c|c|c|c|c|c|c|}
\hline \multirow{2}{*}{ NORMA } & \multicolumn{3}{|c|}{ DIAMETROS } & \multicolumn{2}{c|}{ ALTURAS } & \multicolumn{2}{c|}{ VICAT } \\
\cline { 2 - 8 } & Inicial & Final & $\Delta \%$ & Inicial & Final & $\Delta \%$ & Inicial & Final \\
\hline Francesa & 80,2 & 95,7 & 19,32 & 37,5 & 44,8 & 19,45 & 28 & 3 \\
Acelerada & 80,2 & 81,0 & 1,00 & 40,3 & 41,0 & 1,70 & 0 & 0 \\
\hline
\end{tabular}

Al igual que en los casos anteriores, se facilita fotografías del estado externo de las probetas.

\subsection{Cemento n. ${ }^{\circ} 4$}

\section{Norma Francesa - Pasta \\ 15 - Ettringita. \\ 12 - Hidróxido cálcico.}

\section{Norma Francesa - Pasta desecada}

\section{$\boldsymbol{y}$ - Ettringita.}

12 - Hidróxido cálcico.
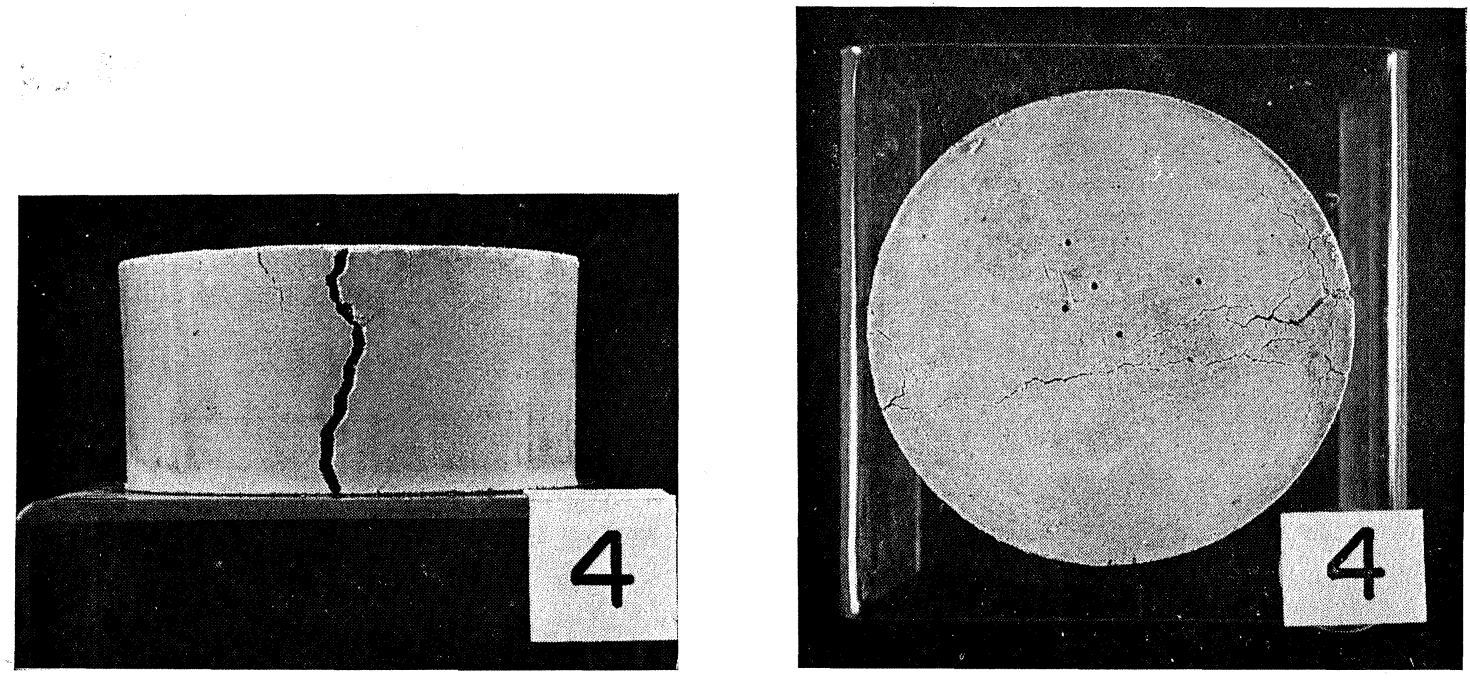

Cemento n.o 4 - Norma Francesa

Comentario: La única variación entre pasta y pasta desecada de la Norma Francesa se debe a la disminución de ettringita aproximadamente a la mitad. En las gráficas de ambas pastas puede apreciarse la poca cristalinidad de la ettringita y del hidróxido cálcico. 

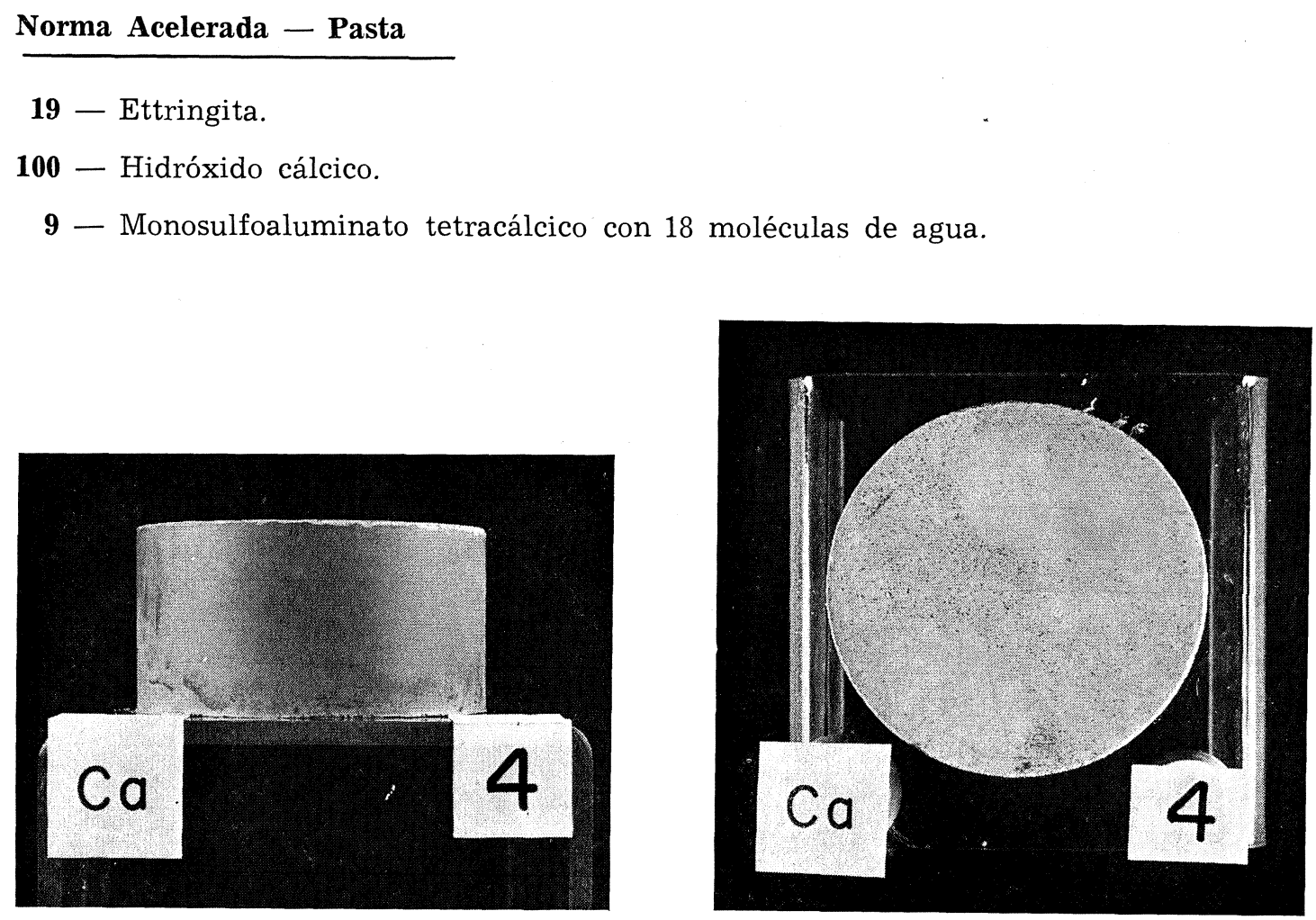

Cemento n.o 4 - Norma Acelerada

Comentario: Siendo la hidratación acelerada más profunda, es explicable el aumento de la cantidad de ettringita y de la cantidad de hidróxido cálcico. La fase monosulfoaluminato tetracálcico con 18 moléculas de agua demuestra una vez más que la hidratación acelerada es más profunda que la llevada a cabo con la Norma Francesa y, por tanto, en ella se darán productos que indican un estado más avanzado de hidratación.

\section{Resultados sobre probetas}

\begin{tabular}{|c|c|c|c|c|c|c|c|c|}
\hline \multirow{2}{*}{ NORMA } & \multicolumn{3}{|c|}{ DIAMETROS } & \multicolumn{2}{c|}{ ALTURAS } & \multicolumn{2}{c|}{ VICAT } \\
\cline { 2 - 8 } & Inicial & Final & $\Delta \%$ & Inicial & Final & $\Delta \%$ & Inicial & Final \\
\hline Francesa & 80,2 & 80,6 & 0,49 & 28,9 & 38,0 & $-2,32$ & 0 & 0 \\
Acelerada & 80,2 & 80,5 & 0,40 & 42,0 & 42,8 & 1,90 & 0 & 0 \\
\hline
\end{tabular}

Se facilitan fotografías del estado externo də las probetas.

\subsection{Cemento $n .^{\circ} 5$}

\section{Norma Francesa - Pasta}

20 - Ettringita.

10 - Yeso.

y - Aluminato tetracálcico con 13 moléculas de agua. 


\begin{abstract}
Y - Monosulfoaluminato tetracálcico con 12 moléculas de agua.
12 - Monosulfoaluminato tetracálcico con 18 moléculas de agua.
\end{abstract}

Comentario: La cantidad de ettringita es mayor que en los cuatro cementos anteriores a causa según Chatterji (10), de que la formación de ettringita en cementos de alto horno, con bajo calor de hidratación, es más rápida que en otros cementos.

La presencia de monosulfoaluminato con 12 moléculas de agua es posible que sea debida, según Chatterji (6), a la reacción entre el aluminato tetracálcico con 13 moléculas de agua y los iones $\mathrm{SO}_{4}=$

El compuesto monosulfoaluminato tetracálcico con 18 moléculas de agua puede existir de una manera estable, según Mehta y Klein (11), como consecuencia de la alta relación $\mathrm{Al}_{2} \mathrm{O}_{3} / \mathrm{SO}_{3}$ y que para este cemento es de 4,13.

\title{
Norma Francesa - Pasta desecada
}
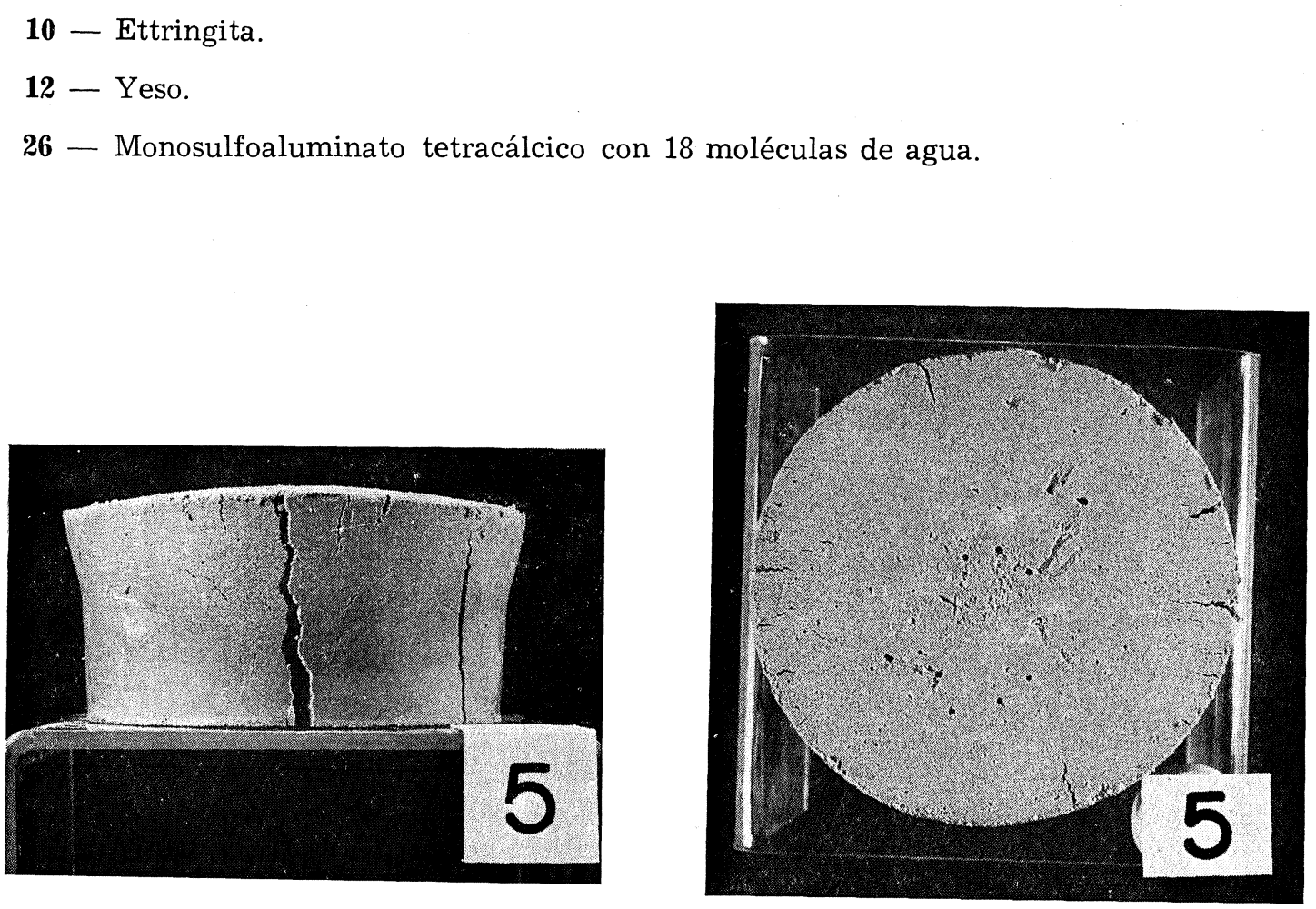

Cemento n.o 5 - Norma Francesa

Comentario: Hay una disminución de ettringita. La cantidad de yeso permanece constante, a causa de que a la temperatura de $40^{\circ} \mathrm{C}$ no hay pérdida de agua. La cantidad de monosulfoaluminato con 18 moléculas de agua aumenta, debido al tiempo prolongado de estancia en estufa ( 17 días) y a que la temperatura de $40^{\circ} \mathrm{C}$ no influye en la pérdida de agua de cristalización. 


$$
\begin{aligned}
& \text { Norma Acelerada - Pasta } \\
& \mathbf{5 0} \text { - Ettringita. } \\
& \mathbf{1 4} \text { - Monosulfoaluminato tetracálcico con } 12 \text { moléculas de agua. } \\
& \mathbf{9} \text { - Monosulfoaluminato tetracálcico con } 18 \text { moléculas de agua. }
\end{aligned}
$$
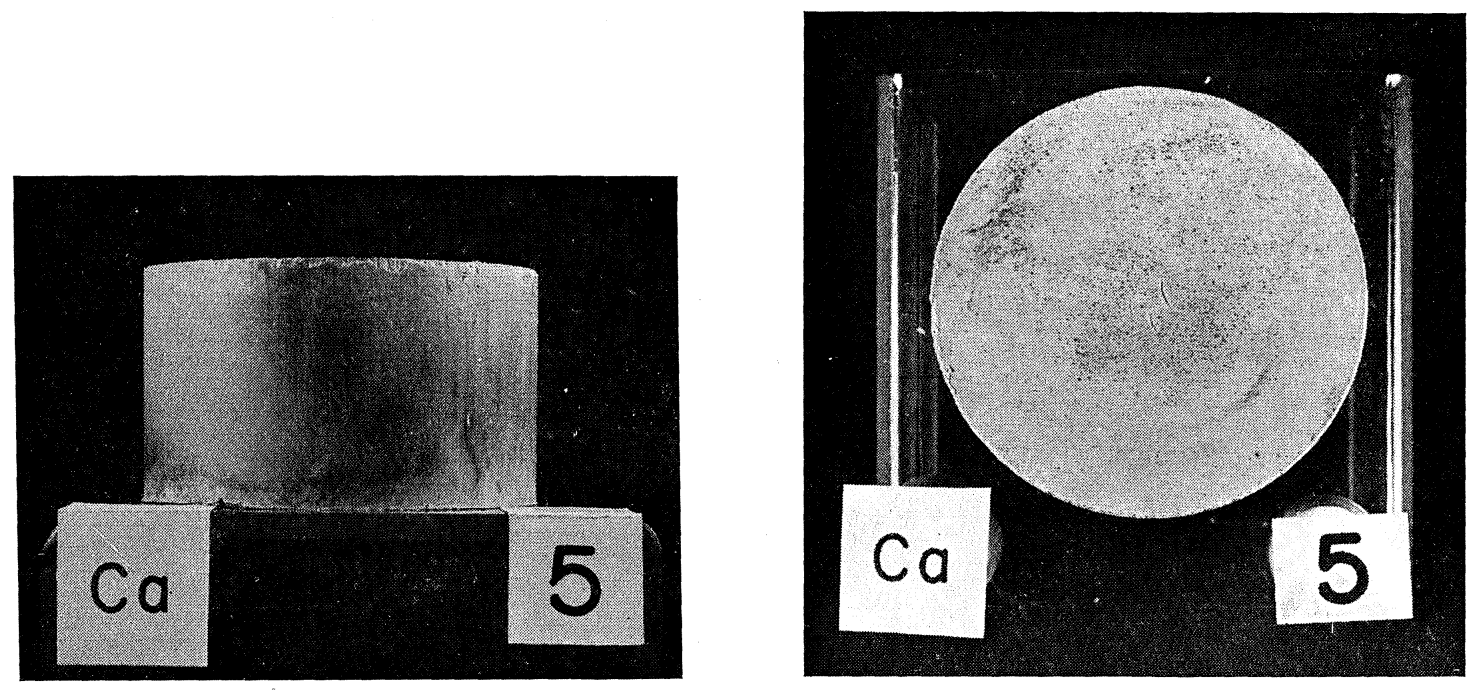

Cemento n.o 5 - Norma acelerada

Comentario: La desaparición del yeso indica el avanzado estado de hidratación. La ettringita aumenta en esta gráfica, a causa del tiempo transcurrido y de la agitación que cataliza la formación de compuestos pertenecientes a un estado avanzado de hidratación. Los cambios dados en las cantidades de monosulfoaluminato tetracálcico con 12 y con 18 moléculas de agua pueden ser producidos por desecaciones sufridas en la manipulación de filtrado, montaje en portamuestras de rayos $\mathrm{X}$ y tiempo de duración del registro de difracción de rayos $\mathrm{X}$.

\section{Resultados sobre probetas}

\begin{tabular}{|c|c|c|c|c|c|c|c|c|}
\hline \multirow{2}{*}{ NORMA } & \multicolumn{3}{|c|}{ DIAMETROS } & \multicolumn{3}{c|}{ ALTURAS } & \multicolumn{2}{c|}{ VICAT } \\
\cline { 2 - 8 } & Inicial & Final & $\Delta \%$ & Inicial & Final & $\Delta \%$ & Inicial & Final \\
\hline Francesa & 80,2 & 91,0 & 13,46 & 38,9 & 42,6 & 9,51 & 0,5 & 0 \\
Acelerada & 80,2 & 80,7 & 0,60 & 48,0 & 48,1 & 0,20 & 0 & 5 \\
\hline
\end{tabular}

Las fotos que a continuación se detallan demuestran, por comparación de ambas normas, la diferencia del estado externo de cada probeta para un mismo cemento. Las probetas de la Norma Francesa presentan gran aumento de volumen y grietas profundas. En cambio, las probetas de la Norma Acelerada permanecen inalteradas en cuanto a dimensiones y carecen de grietas. 


\section{B I B L I O G R A F I A}

(1) Blondiau, L.: “Considérations diverses rélatives à l'essai de la résistance chimique au sulfate de calcium suivant le processus Le Chatelier-Anstett”, Rev. Mat. de Construction, Marzo 1961 , n. 546 pp. 189-200.

Garcia DE Paredes, P.: "Monografía n. ${ }^{\circ}$ 232", del Instituto Eduardo Torroja de la Construcción y del Cemento. Madrid (1963).

(2) Garcia de Paredes, P. y Sagrera, J. L.: "Hidratación acelerada de conglomerantes hidráulicos utilizados en el ensayo de Le Chatelier-Anstett”, Revista ION, Vol. XXX, n. ${ }^{\circ}$ 253, págs. 677-680 (1970).

(3) Garcia de Paredes, P. y Sagrera, J. L.: "Contribución de la difracción de rayos $\mathrm{X}$ al conocimiento de las reacciones entre los conglomerantes hidráulicos y los sulfatos”, Silicates Industriels, XXXVI, n. 6 , págs. 155-160 (1971).

(4) Sagrera, J. L.: "Monografía n.o 295", del Instituto Eduardo Torroja de la Construcción y del Cemento. Madrid (1971).

(5) Metra, P. K.: Journal American Ceramic Society, pp. 204-208 (1967).

(6) Chatterji, S.: Magazine of concrete research, Vol. 19, n. 60 (setiembre 1967).

(7) Kantro, R., and Copeland, T.: Proceedings A.S.T.M., Vol. 60, pp. 1.020 (1960).

(8) Turriziani, R.: L'industria italiana del cemento, n. 12 (diciembre 1968).

(9) Seligmann, P., and Greening, N. R.: Highway Research Record, n. ${ }^{\circ}$ 62, pp. 80 (1964).

(10) Chatterji, S.: Journal American Ceramic Society, pp. 268 (1963).

(11) MEHTA, P. K., and KLEIN, A.: Symposium on structure of portland cement paste and concrete. Special report 90. Highway Research Board (1966). 